\title{
"Rescue" of bilateral subthalamic stimulation by bilateral pallidal stimulation: case report
}

\author{
Caio M. Matias, MD, ${ }^{1,2}$ Danilo Silva, MD, ${ }^{1}$ Andre G. Machado, MD, PhD, ${ }^{1}$ and \\ Scott E. Cooper, MD, PhD1 \\ ${ }^{1}$ Center for Neurological Restoration, Cleveland Clinic Neurological Institute, Cleveland, Ohio; and 2Department of Surgery and \\ Anatomy, Ribeirão Preto Medical School, University of São Paulo, Ribeirão Preto, São Paulo, Brazil
}

\begin{abstract}
Deep brain stimulation (DBS) of the subthalamic nucleus (STN) or globus pallidus pars interna (GPi) is well established as a treatment for advanced Parkinson's disease. In general, one of the 2 targets is chosen based on the clinical features of each patient. Stimulation of both targets could be viewed as redundant, given that the 2 targets are directly connected. However, it is possible that each target has different mechanisms, with clinical effects mediated by orthodromic or antidromic stimulation.

The authors report the case of a patient with severe Parkinson's disease who had previously undergone bilateral subthalamic stimulation with excellent benefits. However, he presented with significant worsening associated with disease progression and pharmacological treatment, and then underwent bilateral GPi DBS. Follow-up assessment was conducted clinically as well as through blinded ratings of video recordings.

Pallidal DBS may be a safe and useful strategy to manage dystonic features and behavioral complications of subthalamic stimulation and pharmacological management. While combined stimulation was quite successful in the reported patient, further studies with larger samples and longer follow-up periods will be necessary before recommending the addition of pallidal DBS as a routine strategy for patients previously implanted with STN DBS.
\end{abstract}

http://thejns.org/doi/abs/10.3171/2015.1.JNS141604

KEY WORDS deep brain stimulation; dual target; globus pallidus; Parkinson's disease; subthalamic nucleus; functional neurosurgery

$\mathrm{D}$ EEP brain stimulation (DBS) is a standard treatment for selected patients with advanced Parkinson's disease (PD)., 4, $, 7,15,17,21,26$ Randomized controlled studies have shown that DBS combined with medical therapy provides superior outcomes compared with medical management alone for the control of motor symptoms in advanced disease. . $, 33,36$ There are currently 3 major DBS targets for managing PD symptoms. While thalamic stimulation is usually reserved for a few patients with tremor-predominant $\mathrm{PD},{ }^{18,27}$ subthalamic nucleus (STN) and globus pallidus pars interna (GPi) DBS can treat motor cardinal symptoms of the disease, including tremor, rigidity, and bradykinesia, and manage motor fluctuations. ${ }^{6}$
Randomized comparisons between GPi and STN DBS showed similar outcomes for both nuclei. ${ }^{9,14,34}$ Concerns related to higher hemorrhage risk during microelectrode recording-guided GPi DBS surgery ${ }^{2}$ can make this a lessfavored target by some centers. However, cognitive and behavioral adverse effects may be more common after STN DBS. ${ }^{9,34}$ In our center, we consider patients for both STN and GPi surgery and tailor the target choice to the patient's goals and presentation. Although DBS is effective for symptom management, it does not prevent the progression of the disease. ${ }^{5,8}$ We present here the case of a patient in whom bilateral STN DBS was effective, but whose PD progressed with severe motor, particularly dystonic,

ABBREVIATIONS DBS = deep brain stimulation; GPi = globus pallidus pars interna; IPG = implantable pulse generator; LEDD = levodopa equivalent daily dose; MDSUPDRS = Movement Disorder Society-Sponsored Revision of the Unified Parkinson's Disease Rating Scale; NRS-11 = 0- to 10-point Numerical Rating Scale; PD = Parkinson's disease; $\mathrm{PW}=$ pulse width; $\mathrm{STN}=$ subthalamic nucleus.

SUBMITTED July 8, 2014. ACCEPTED January 21, 2015.

INCLUDE WHEN CITING Published online July 31, 2015; DOI: 10.3171/2015.1.JNS141604.

DISCLOSURE Dr. Machado reports that he is a patent holder with Enspire, ATI, and Cardionomics; that he is a consultant for Functional Neuromodulation, Spinal Modulation, and Icahn; and that Medtronic supports the fellowship program at his center. He also reports that NIH supports his research (not related to this manuscript), including percent effort of his salary. Dr. Matias received a scholarship from the CAPES Foundation, Ministry of Education of Brazil, Brasília -DF 70.040-020, Brazil. Process number Bex 2536/13-7. 
symptoms after 12 years that could not be managed with STN DBS alone. He underwent bilateral implantation of additional GPi DBS leads with good motor benefits.

\section{Case Report}

We present the case of a 41-year-old man with an 8-year history of PD who was experiencing progressive disability despite optimal medical therapy. His major complaints were freezing of gait, rigidity, unpredictable off-periods, and severe dyskinesia. He underwent bilateral STN DBS and his symptoms were markedly improved, except for some residual mild bradykinesia on the left hand. At the 1-year follow-up, the levodopa equivalent daily dose $(\mathrm{LEDD})^{31}$ of $2150 \mathrm{mg}$ was reduced to $750 \mathrm{mg}$ (-65\%) (Fig. 1). Symptom control remained stable for 4 years after surgery, but as the disease progressed, the patient started to complain of left arm dystonia, initially only with strenuous exercise such as participating in a triathlon, which he continued to do annually. This was initially managed with botulinum toxin injections, but the left arm dystonia worsened, affecting other parts of the body and becoming painful, with an average pain reporting of 7 on a 0 - to 10-point Numerical Rating Scale (NRS-11). ${ }^{37}$ Hardware failure was ruled out, and several trials to adjust stimulation parameters failed to improve his condition. Stimulation amplitude was increased until side effects were noted at $4.1 \mathrm{~V}$ and above, with worsening of left arm cramping. Although dopaminergic treatment and stimulation were still beneficial, the patient could not tolerate further amplitude or medication increases. In addition, he experienced worsening on-medication festination of speech and gait, the latter leading to falls. Moreover, he developed severe neuropsychological problems including inappropriate sexual behavior and impulsive spending, which caused significant financial, interpersonal, and family problems. With reduction in total daily levodopa equivalent (dopamine agonists were reduced by $55 \%$ ), the neuropsychological symptoms resolved, but at the cost of motor worsening and marked dystonic postures.

At the age of 53 years (12 years after the first surgery), the patient's quality of life was assessed using the EQ-5D, ${ }^{25}$ yielding an index score of 0.378 , indicative of poor health status (the EQ-5D index score ranges from -0.109 to 1.0 , in which a higher score indicates a better quality of life). Activities of daily living were assessed using the Movement Disorder Society-Sponsored Revision of the Unified Parkinson's Disease Rating Scale (MDS-UPDRS) ${ }^{10}$ Part II (score range 0-52), yielding a score of 25. Motor function was evaluated by an independent rater blinded to stimulation and medication condition with the MDS-UPDRS Part III (score range 0-112) during the on-medication/ on-stimulation state, also yielding a score of 25 points. By the time of surgical consideration, the patient was presenting with dystonic symptoms involving the trunk and both sides. A decision was made to add bilateral GPi electrodes with the goal of treating these symptoms, but the patient did not believe he could tolerate an awake procedure again, particularly due to severe dystonic symptoms that could be worsened in a supine, fixed position. GPi electrodes were implanted under general anesthesia, with placement verification by intraoperative images with the $\mathrm{O}$-arm (Medtronic Inc.) fused with the preoperative images and surgical plan in the StealthStation (Medtronic Inc.). New implantable pulse generators (IPGs) were implanted and connected to the leads in a second stage. One month after the pallidal leads were implanted, the IPGs were turned on. Stimulation parameters previously used for the STN were maintained (left STN: bipolar $=1-2-3+$, amplitude $=4.0$ $\mathrm{V}$, pulse width $[\mathrm{PW}]=120 \mu \mathrm{sec}$, rate $=130 \mathrm{~Hz}$; right STN: bipolar $=1-2-3+$, amplitude $=4.3 \mathrm{~V}, \mathrm{PW}=90 \mu \mathrm{sec}$, rate $=$ $130 \mathrm{~Hz}$ ). Pallidal stimulation parameters were set as monopolar initially (left GPi: monopolar $=$ case $+10-$, amplitude $=1.5 \mathrm{~V}, \mathrm{PW}=60 \mu \mathrm{sec}$, rate $=130 \mathrm{~Hz}$; right GPi: monopolar $=$ case $+8-$, amplitude $=2.3 \mathrm{~V}, \mathrm{PW}=60 \mu \mathrm{sec}$, rate $=$ $130 \mathrm{~Hz}$ ), but were gradually titered during follow-up visits. Stimulation parameters at the 6-month follow-up and stereotactic coordinates for both targets are presented in Table 1. Six months after surgery, motor outcome during onmedication/on-stimulation (STN and GPi) state measured by blinded ratings of MDS-UPDRS Part III was improved by $60 \%$, mostly due to improvement in kinetic tremor and bradykinesia, activities of daily living (MDS-UPDRS Part II) by $44 \%$, pain (NRS-11) by $42 \%$, and quality of life by $82 \%$ according to the EQ-5D (Table 2). The LEDD was further reduced, by $55 \%$ (Fig. 1). Images of subthalamic and pallidal electrodes are displayed in Fig. 2.

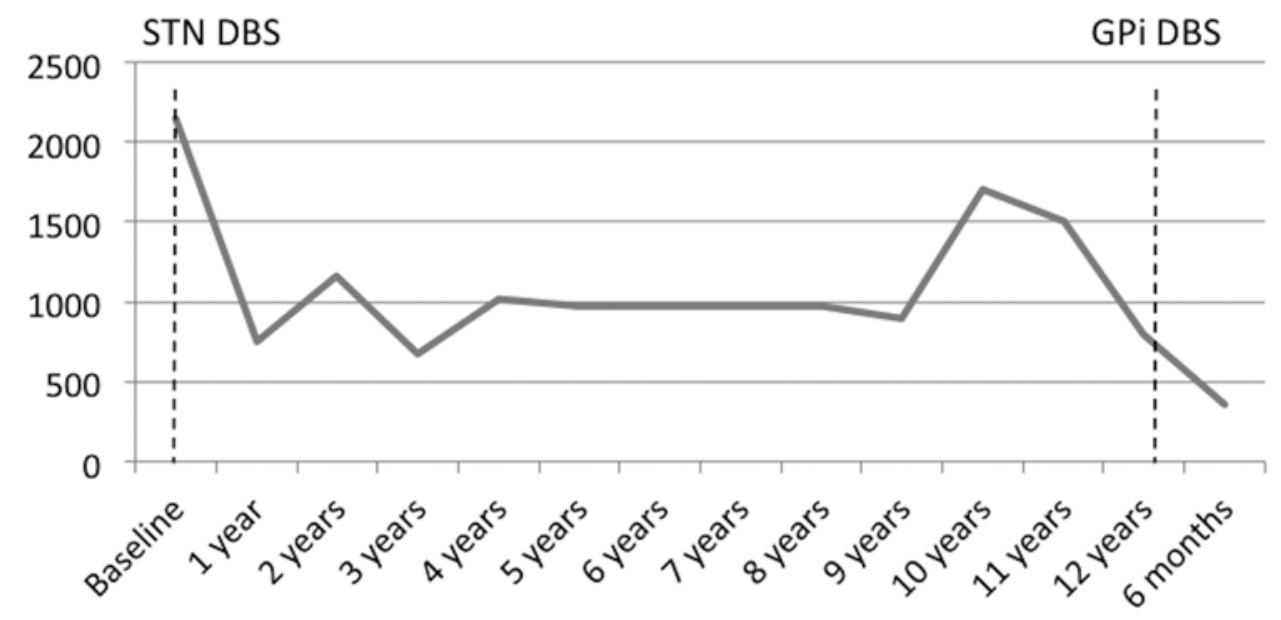

FIG. 1. LEDD (in mg) over time. 
TABLE 1. Electrodes, stereotactic coordinates, and stimulation parameters 6 months after pallidal electrode implantation

\begin{tabular}{|c|c|c|c|c|c|c|c|}
\hline \multirow[b]{2}{*}{ Location } & \multicolumn{3}{|c|}{ Stereotactic Coordinates* } & \multicolumn{4}{|c|}{ Stimulation Parameters } \\
\hline & $x$ & $Y$ & Z & Amplitude (V) & PW ( $\mu s e c)$ & Rate $(\mathrm{Hz})$ & Configuration \\
\hline \multicolumn{8}{|l|}{ STN } \\
\hline Right & +11.5 & -2.8 & -6.7 & 4.3 & 90 & 130 & $1-2-3+$ \\
\hline Left & -12.3 & -3.9 & -6.7 & 4.0 & 120 & 130 & $1-2-3+$ \\
\hline \multicolumn{8}{|l|}{ GPi } \\
\hline Right & +25.3 & +3.4 & -7.5 & 3.6 & 60 & 130 & $8-9-C+$ \\
\hline Left & -24.4 & +2.1 & -6.3 & 3.6 & 60 & 130 & $10-11-C+$ \\
\hline
\end{tabular}

* Tip of the electrode relative to the midcommissural point (mm).

\section{Discussion}

Our report describes a patient who experienced significant benefit for several years following STN DBS. However, behavioral problems and painful dystonia gradually emerged over time. Impulse control disorders can be associated with long-term dopaminergic treatment, particularly dopamine agonists. ${ }^{3}$ Weintraub et al. ${ }^{35}$ reported an overall impulse control disorder prevalence of $13.6 \%$ in PD patients receiving dopamine-replacement therapy (compulsive sexual behavior, 3.5\%; pathological gambling, 5.0\%; compulsive buying, 5.7\%; binge-eating disorder, $4.3 \% ; 2$ or more impulse control disorders combined, 3.9\%). Decreasing dopamine agonists results in a less pulsatile dopaminergic stimulation and is associated with remission of or significant reduction in impulse control disorder. ${ }^{19}$ Likewise, dystonia can occur as a complication of medical therapy in PD patients..$^{24,30}$ It usually occurs as an offperiod phenomenon and the lower limb, particularly the foot, is the most commonly affected location. ${ }^{24}$ However, it can also present as peak dose dystonia or diphasic dystonia. There are different approaches to control dystonia, including several pharmacological strategies, botulinum toxin injections, and DBS. ${ }^{30}$ Levodopa dose reduction or withdrawal usually improves "on" time dystonia, but other parkinsonian symptoms may worsen. In our case, neuropsychological problems resolved with reduction of dopamine agonists, but motor function worsened significantly. Moreover, dystonia became severely painful despite botulinum toxin injections. As subthalamic stimulation was still effective, we concluded that these leads should not be removed and replaced with pallidal leads. Rather, addition-

TABLE 2. Clinical outcomes 6 months after bilateral STN and bilateral GPi stimulation according to different rating scales

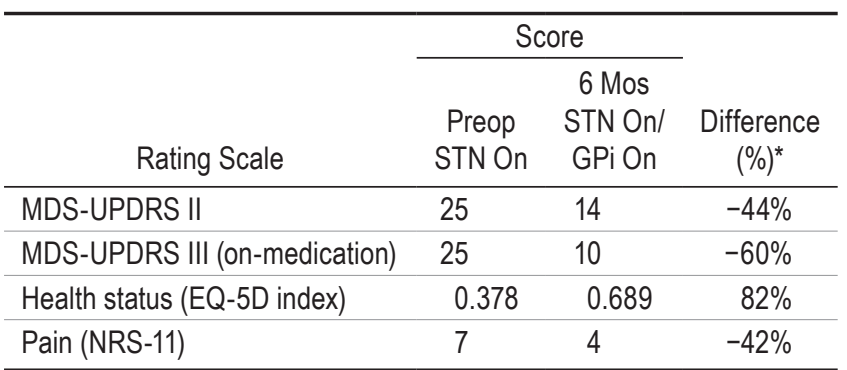

* Negative difference indicates improvement, except for EQ-5D index, for which a positive difference indicates improvement. al pallidal leads were implanted with the goal of improving motor function and dystonic symptoms.

According to the "classic" theory of how STN DBS works, stimulation orthodromically activates efferent axons. Adding pallidal electrodes might therefore be considered redundant and unlikely to add benefits in managing symptoms because subthalamic stimulation already influences GPi activity via the subthalamopallidal projection. ${ }^{12}$ However, recent work by Gradinaru et al. ${ }^{11}$ and $\mathrm{Li}$ et al. ${ }^{16}$ suggested that antidromic activation of afferents to the STN may contribute to DBS therapeutic effects. According to this view, GPi DBS might activate physiological mechanisms different from those of STN DBS, justifying the addition of GPi DBS in a patient such as ours. In de-

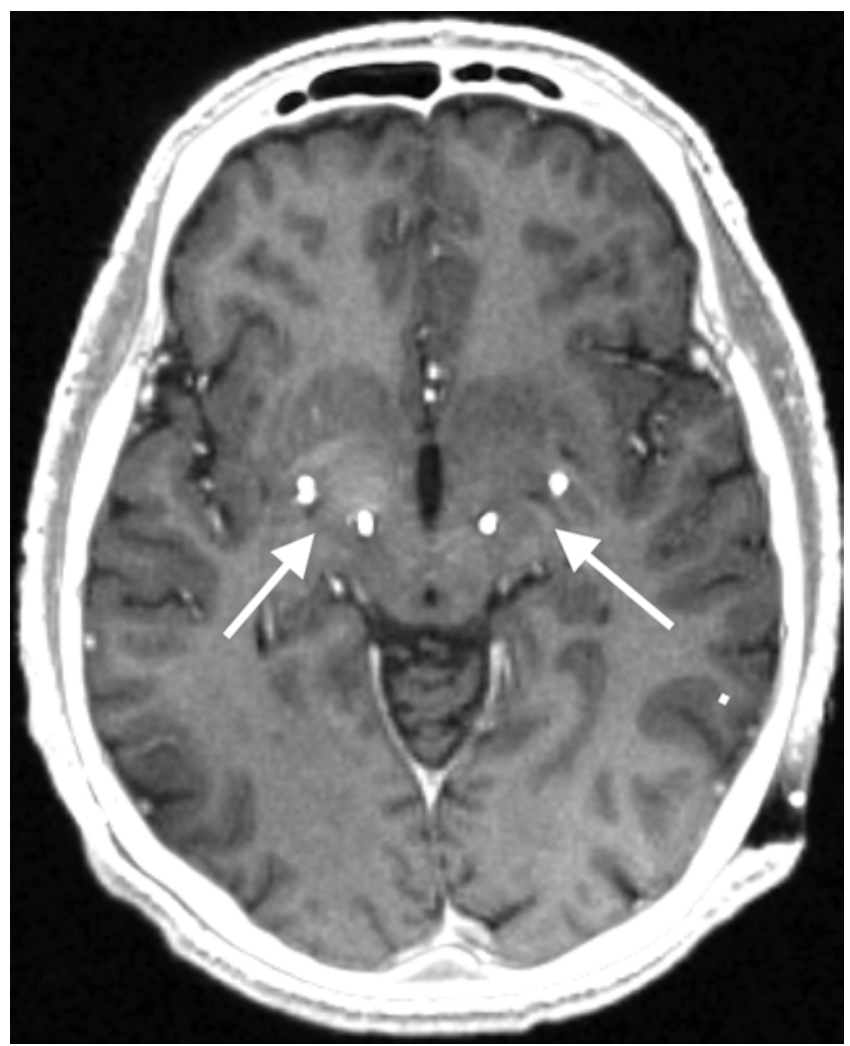

FIG. 2. Preoperative MR images were fused with postoperative CT. Subthalamic electrodes (located medially) and pallidal electrodes (located laterally) are indicated by the arrows. 
ciding whether to offer GPi DBS to this patient, we also considered another, simpler rationale. Targeting DBS in PD patients aims at stimulating as much of the motor corticobasal ganglia-thalamo-cortical loop ${ }^{13}$ as possible while minimizing spread of current to limbic or associative loops, or to adjacent structures such as the internal capsule. ${ }^{20,32}$ Adding GPi DBS may allow for stimulation of additional motor fibers, which could not be captured from STN alone without spread of current to other undesired structures. A third rationale for adding GPi DBS in our patient was the prominence of dystonia among his symptoms. Clinical observation has suggested that pallidal stimulation may be beneficial for dystonic symptoms, ${ }^{28}$ although subthalamic stimulation has recently been shown to also be effective for primary generalized and segmental dystonia. ${ }^{22,28}$

Prior reports have studied the possibility of co-targeting STN and GPi in patients with PD. Peppe et al. ${ }^{23}$ reported no difference between subthalamic stimulation alone versus combined stimulation in 14 patients with PD. Allert et al. reported the case of a patient with PD in whom additional pallidal stimulation successfully corrected the failure of long-term subthalamic stimulation. ${ }^{1}$ Two major differences contrast our report from the former study. In the case of Peppe et al., all 4 electrodes had been implanted on the same day to directly compare the initial effects of singleand dual-target stimulation. In our patient, pallidal electrodes were implanted after 12 years to attempt to rescue long-term efficacy decline and complications associated with subthalamic stimulation. This difference points out the possibility that indeed there may be little advantage to simultaneously target the subthalamus and pallidum, but an opportunity exists for rescuing long-term limitations of subthalamic stimulation with pallidal leads. Second, the mean disease duration in the report by Peppe et al. was 13 years, while in our case the disease duration was 20 years at the time of surgery. Similar to our case, Allert and colleagues reported on a patient with young-onset PD in whom subthalamic stimulation initially improved motor function. However, in that case, symptom control declined faster and motor function was significantly impaired 4 years after subthalamic stimulation, whereas in our case significant impairment occurred almost a decade later.

Although the present report has strengths, such as blinded UPDRS ratings before and after implantation of pallidal leads, it also has limitations. First of all, it consists of a single-patient report and thus caution must be taken before generalizing this treatment to other patients. Furthermore, our case may not represent an ordinary case of idiopathic PD due to features such as young onset, rapid progression to motor fluctuation, and prominent dystonia, which can be associated with some genetic forms of parkinsonism. ${ }^{29} \mathrm{Un}$ fortunately, this patient has not been submitted to genetic testing to exclude a genetic etiology. In addition, it was difficult to obtain on-/off-medication and on-/off-stimulation assessments separately for GPi, STN, and both together. The patient travels a considerable distance to attend our clinic and would have to stay for an overnight medication washout. Furthermore, testing all combinations of stimulation, with time allowed for washout, would require very prolonged testing in an unmedicated and unstimulated or partially stimulated state. In that state, this patient devel- ops a very deep off-stimulation and off-medication severe, painful dystonia. The patient previously agreed to limited testing of this kind, for purposes of clinical care, but we did not feel justified in performing more difficult and uncomfortable testing to provide novel scientific information. Moreover, the patient gave permission for videotapes to be made for clinical and scientific purposes, but he did not give permission for them to be published. Nevertheless, we were able to assess outcome measures with reliable and well-established tools such as EQ-5D, MDS-UPDRS Part II, and blinded MDS-UPDRS Part III assessments. Sixty percent is an exceptionally large improvement in medicated motor UPDRS score and may reflect several factors specific to this patient. The figure is not the average of a sample but rather for an individual patient and may therefore come from the tail of the statistical distribution. The likelihood that this case represents the tail of distribution is emphasized by patient selection. This patient was not randomly selected: to the contrary, he represents a very successful case of our center, and this influenced our decision to offer supplemental GPi electrodes. Some individual characteristics may also explain his above-average response to DBS: his comparatively young age, his young age at onset, and very prominent off-medication and offstimulation dystonia. Moreover, he is passionately devoted to physical exercise (for example, he continued to exercise heavily until shortly before the GPi surgery). In addition, his on-medication UPDRS score may have been lowered by the medication reduction necessitated in his case by neuropsychiatric drug side effects.

In the past few years, outcomes of patients with PD after 8-10 years of subthalamic DBS have been reported, ${ }^{5,8,38}$ and the number of patients who have undergone long-term subthalamic stimulation is expected to increase. Managing symptoms in this population and identifying therapeutic approaches that might address limitations and complications of chronic subthalamic stimulation will become increasingly important. Pallidal DBS may be a safe and useful strategy to manage dystonic features and behavioral complications of subthalamic stimulation and pharmacological management. While combined stimulation was quite successful in the reported patient, further studies with larger samples and longer follow-up periods will be necessary before recommending the addition of pallidal DBS as a routine strategy for patients previously implanted with STN DBS.

\section{Acknowledgment}

We thank Srivadee Oravivattanakul, MD, for her support with the blinded MDS-UPDRS Part III evaluations.

\section{References}

1. Allert N, Schnitzler A, Sturm V, Maarouf M: Failure of longterm subthalamic nucleus stimulation corrected by additional pallidal stimulation in a patient with Parkinson's disease. J Neurol 259:1244-1246, 2012

2. Binder DK, Rau G, Starr PA: Hemorrhagic complications of microelectrode-guided deep brain stimulation. Stereotact Funct Neurosurg 80:28-31, 2003

3. Broen M, Duits A, Visser-Vandewalle V, Temel Y, Winogrodzka A: Impulse control and related disorders in Parkinson's disease patients treated with bilateral subthalamic 
nucleus stimulation: a review. Parkinsonism Relat Disord 17:413-417, 2011

4. Brown AW, Marlowe KJ, Bjelke B: Age effect on motor recovery in a post-acute animal stroke model. Neurobiol Aging 24:607-614, 2003

5. Castrioto A, Lozano AM, Poon YY, Lang AE, Fallis M, Moro E: Ten-year outcome of subthalamic stimulation in Parkinson disease: a blinded evaluation. Arch Neurol 68: 1550-1556, 2011

6. Deep-Brain Stimulation for Parkinson's Disease Study Group: Deep-brain stimulation of the subthalamic nucleus or the pars interna of the globus pallidus in Parkinson's disease. N Engl J Med 345:956-963, 2001

7. Deuschl G, Schade-Brittinger C, Krack P, Volkmann J, Schäfer H, Bötzel K, et al: A randomized trial of deep-brain stimulation for Parkinson's disease. N Engl J Med 355:896-908, 2006

8. Fasano A, Romito LM, Daniele A, Piano C, Zinno M, Bentivoglio AR, et al: Motor and cognitive outcome in patients with Parkinson's disease 8 years after subthalamic implants. Brain 133:2664-2676, 2010

9. Follett KA, Weaver FM, Stern M, Hur K, Harris CL, Luo P, et al: Pallidal versus subthalamic deep-brain stimulation for Parkinson's disease. N Engl J Med 362:2077-2091, 2010

10. Goetz CG, Tilley BC, Shaftman SR, Stebbins GT, Fahn S, Martinez-Martin P, et al: Movement Disorder Societysponsored revision of the Unified Parkinson's Disease Rating Scale (MDS-UPDRS): scale presentation and clinimetric testing results. Mov Disord 23:2129-2170, 2008

11. Gradinaru V, Mogri M, Thompson KR, Henderson JM, Deisseroth $\mathrm{K}$ : Optical deconstruction of parkinsonian neural circuitry. Science 324:354-359, 2009

12. Hashimoto T, Elder CM, Okun MS, Patrick SK, Vitek JL: Stimulation of the subthalamic nucleus changes the firing pattern of pallidal neurons. J Neurosci 23:1916-1923, 2003

13. Kelly RM, Strick PL: Macro-architecture of basal ganglia loops with the cerebral cortex: use of rabies virus to reveal multisynaptic circuits. Prog Brain Res 143:449-459, 2004

14. Kopell BH, Halverson J, Butson CR, Dickinson M, Bobholz $\mathrm{J}$, Harsch H, et al: Epidural cortical stimulation of the left dorsolateral prefrontal cortex for refractory major depressive disorder. Neurosurgery 69:1015-1029, 2011

15. Krack P, Batir A, Van Blercom N, Chabardes S, Fraix V, Ardouin $\mathrm{C}$, et al: Five-year follow-up of bilateral stimulation of the subthalamic nucleus in advanced Parkinson's disease. $\mathbf{N}$ Engl J Med 349:1925-1934, 2003

16. Li Q, Ke Y, Chan DC, Qian ZM, Yung KK, Ko H, et al: Therapeutic deep brain stimulation in Parkinsonian rats directly influences motor cortex. Neuron 76:1030-1041, 2012

17. Limousin P, Krack P, Pollak P, Benazzouz A, Ardouin C, Hoffmann D, et al: Electrical stimulation of the subthalamic nucleus in advanced Parkinson's disease. N Engl J Med 339:1105-1111, 1998

18. Lyons KE, Koller WC, Wilkinson SB, Pahwa R: Long term safety and efficacy of unilateral deep brain stimulation of the thalamus for parkinsonian tremor. J Neurol Neurosurg Psychiatry 71:682-684, 2001

19. Mamikonyan E, Siderowf AD, Duda JE, Potenza MN, Horn S, Stern MB, et al: Long-term follow-up of impulse control disorders in Parkinson's disease. Mov Disord 23:75-80, 2008

20. Manola L, Roelofsen BH, Holsheimer J, Marani E, Geelen J: Modelling motor cortex stimulation for chronic pain control: electrical potential field, activating functions and responses of simple nerve fibre models. Med Biol Eng Comput 43:335-343, 2005

21. Moro E, Lozano AM, Pollak P, Agid Y, Rehncrona S, Volkmann J, et al: Long-term results of a multicenter study on subthalamic and pallidal stimulation in Parkinson's disease. Mov Disord 25:578-586, 2010
22. Ostrem JL, Racine CA, Glass GA, Grace JK, Volz MM, Heath SL, et al: Subthalamic nucleus deep brain stimulation in primary cervical dystonia. Neurology 76:870-878, 2011

23. Peppe A, Pierantozzi M, Bassi A, Altibrandi MG, Brusa L, Stefani A, et al: Stimulation of the subthalamic nucleus compared with the globus pallidus internus in patients with Parkinson disease. J Neurosurg 101:195-200, 2004

24. Poewe WH, Lees AJ, Stern GM: Dystonia in Parkinson's disease: clinical and pharmacological features. Ann Neurol 23:73-78, 1988

25. Rabin R, de Charro F: EQ-5D: a measure of health status from the EuroQol Group. Ann Med 33:337-343, 2001

26. Rodriguez-Oroz MC, Obeso JA, Lang AE, Houeto JL, Pollak $\mathrm{P}$, Rehncrona S, et al: Bilateral deep brain stimulation in Parkinson's disease: a multicentre study with 4 years follow-up. Brain 128:2240-2249, 2005

27. Savica R, Matsumoto JY, Josephs KA, Ahlskog JE, Stead M, Lee KH, et al: Deep brain stimulation in benign tremulous parkinsonism. Arch Neurol 68:1033-1036, 2011

28. Schjerling L, Hjermind LE, Jespersen B, Madsen FF, Brennum J, Jensen SR, et al: A randomized double-blind crossover trial comparing subthalamic and pallidal deep brain stimulation for dystonia. J Neurosurg 119:1537-1545, 2013

29. Schrag A, Schott JM: Epidemiological, clinical, and genetic characteristics of early-onset parkinsonism. Lancet Neurol 5:355-363, 2006

30. Tolosa E, Compta Y: Dystonia in Parkinson's disease. J Neurol 253 (Suppl 7):VII7-VII13, 2006

31. Tomlinson CL, Stowe R, Patel S, Rick C, Gray R, Clarke CE: Systematic review of levodopa dose equivalency reporting in Parkinson's disease. Mov Disord 25:2649-2653, 2010

32. Tsubokawa T, Katayama Y, Yamamoto T, Hirayama T, Koyama S: Chronic motor cortex stimulation for the treatment of central pain. Acta Neurochir Suppl (Wien) 52:137-139, 1991

33. Weaver FM, Follett K, Stern M, Hur K, Harris C, Marks WJ Jr, et al: Bilateral deep brain stimulation vs best medical therapy for patients with advanced Parkinson disease: a randomized controlled trial. JAMA 301:63-73, 2009

34. Weaver FM, Follett KA, Stern M, Luo P, Harris CL, Hur $\mathrm{K}$, et al: Randomized trial of deep brain stimulation for Parkinson disease: thirty-six-month outcomes. Neurology 79:55-65, 2012

35. Weintraub D, Koester J, Potenza MN, Siderowf AD, Stacy M, Voon V, et al: Impulse control disorders in Parkinson disease: a cross-sectional study of 3090 patients. Arch Neurol 67:589-595, 2010

36. Williams A, Gill S, Varma T, Jenkinson C, Quinn N, Mitchell R, et al: Deep brain stimulation plus best medical therapy versus best medical therapy alone for advanced Parkinson's disease (PD SURG trial): a randomised, open-label trial. Lancet Neurol 9:581-591, 2010

37. Williamson A, Hoggart B: Pain: a review of three commonly used pain rating scales. J Clin Nurs 14:798-804, 2005

38. Zibetti M, Merola A, Rizzi L, Ricchi V, Angrisano S, Azzaro C, et al: Beyond nine years of continuous subthalamic nucleus deep brain stimulation in Parkinson's disease. Mov Disord 26:2327-2334, 2011

\section{Author Contributions}

Conception and design: Matias, Cooper. Acquisition of data: Matias. Drafting the article: Matias, Silva, Cooper. Critically revising the article: all authors.

\section{Correspondence}

Caio M. Matias, Center for Neurological Restoration, Cleveland Clinic Neurological Institute, 9500 Euclid Ave., Cleveland, OH 44195. email: caiomatias@usp.br. 